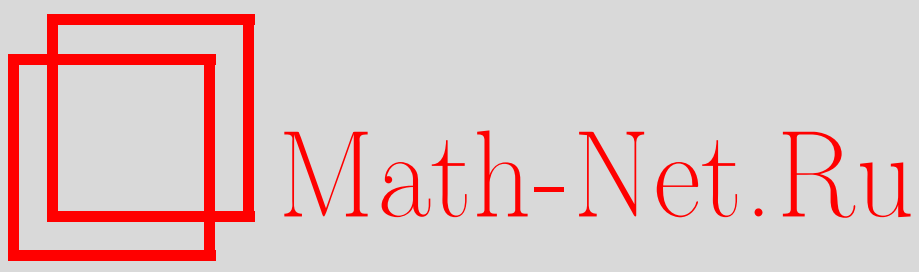

Е. С. Жуковский, Неравенства Вольтерра в функциональных пространствах, $M a$ тем. сб., 2004, том 195, номер 9, 3-18

DOI: https://doi.org/10.4213/sm842

Использование Общероссийского математического портала Math-Net.Ru подразумевает, что вы прочитали и согласны с пользовательским соглашением

http://www . mathnet.ru/rus/agreement

Параметры загрузки:

IP: 52.23 .180 .231

26 апреля 2023 г., 18:11:57 
УДК 517.929

\author{
Е.С. Жуковский
}

\title{
Неравенства Вольтерра в функциональных пространствах
}

\begin{abstract}
Предлагаются утверждения о локальной разрешимости, продолжаемости решений, существовании верхнего и нижнего решений уравнений с монотонными обобщенно вольтерровьми операторами в банаховых функциональных пространствах. Эти утверждения аналогичны известньм теоремам об интегральном и дифференциальном неравенстве и могут использоваться для оценки решений различных функционально-дифференциальных уравнений.
\end{abstract}

Библиография: 31 название.

Важнейшим инструментом исследования уравнений, решение которых нельзя записать явно, являются утверждения о неравенствах, позволяющие строить оценки решений. Классические результаты С.А. Чаплыгина [1] положили начало исследованиям дифференциальных и интегральных неравенств, их широкому применению в теории обыкновенных дифференциальных уравнений. Отметим известные работы Н.В. Азбелева, З.Б. Цалюка [2], [3] и книгу [4], содержащую достаточно полную библиографию. Развитие теории функционально-дифференциальных уравнений потребовало изучения операторных неравенств в различных функциональных пространствах [5], [6]. В [7], [8] получены подобные утверждения для пространства суммируемых на $[a, b]$ функций, упорядоченного “естественным" конусом неотрицательных функций. Работа [9] посвящена обобщению этих результатов на случай произвольного конуса, порождающего “вольтеррову полуупорядоченность" в пространстве суммируемых функций. Здесь мы рассмотрим вольтерровы операторные неравенства в произвольных банаховых пространствах. Необходимость в таких результатах отмечается при изучении сингулярных уравнений, импульсных систем, “гибридных" задач и т.д. [5], [6], [10]-[12]. При этом авторы обычно рассматривают конкретные типы уравнений, указывая на сложность проблемы оценки решений абстрактных уравнений и отсутствие работ по этой тематике [6]. В предлагаемых нами утверждениях не требуется непрерьвность операторов, что позволяет применять полученные результаты к изучению дифференциальных уравнений с разрьвной правой частью, функционально-дифференциальных уравнений с авторегулированием, функционально-дифференциальных уравнений с максимумами и др. Подобньми уравнениями описываются некоторые задачи экономики, биологии, управления, автоматического регулирования [13]-[16]. Неприменимость классических методов, использующих непрерывность операторов, и многочисленные приложения привлекли в последнее десятилетие к таким уравнениям внимание исследователей.

Работа вьполнена при поддержке Российского фонда фундаментальных исследований (грант № 01-01-00140) и Министерства образования РФ (грант № Е02-1.0-212). 
В работе используются следуюшие пространства функций $x:[a, b] \rightarrow \mathbb{R}:$ пространство $C_{[a, b]}$ непрерывных функций, $\|x\|_{C_{[a, b]}}=\max _{t \in[a, b]}|x(t)|$; пространство $L_{[a, b]}$ суммируемых функций, $\|x\|_{L_{[a, b]}}=\int_{a}^{b}|x(t)| d t$; пространство $D_{[a, b]}$ абсолютно непрерывных функций, имеющих производную $x^{\prime} \in L_{[a, b]},\|x\|_{D_{[a, b]}}=$ $\left\|x^{\prime}\right\|_{L_{[a, b]}}+|x(a)|$.

1. Вначале приведем некоторые понятия теории конусов в банаховых пространствах [17], играющие важную роль в нашем исследовании.

Пусть $B$ - банахово пространство. Замкнутое выпуклое множество $\Lambda \subset B$ называют конусом, если из $x \in \Lambda, x \neq 0$, вытекает, что $\lambda x \in \Lambda$ при $\lambda>0$ и $\lambda x \notin \Lambda$ при $\lambda<0$. Для $x, y \in B$ будем писать $x \triangleright y$ или $y \triangleleft x$, если $x-y \in \Lambda$.

Элемент $\bar{z} \in B$ называют точной верхней границей множества $U \subset B$ и пишут $\bar{z}=\sup U$, если для всех $u \in U$ выполнено $\bar{z} \triangleright u$ и для любого $y \in B$ из $y \triangleright u \forall u \in U$ следует $y \triangleright \bar{z}$. Аналогично определяется точная нижняя граница. Если у множества из двух элементов есть точная верхняя (нижняя) граница, то существует и нижняя (верхняя), причем вьполнено равенство $\sup \{x, y\}+$ $\inf \{x, y\}=x+y$. Конус $\Lambda \subset B$ называют миниэдральнылм, если у каждого множества из двух элементов есть точная нижняя (и, следовательно, верхняя) граница. Каждый элемент $x \in B$ в случае миниэдральности конуса $\Lambda$ допускает представление $x=x_{+}+x_{-}$, где $x_{+}=\sup \{x, 0\} \in \Lambda, x_{-}=\sup \{-x, 0\} \in \Lambda$. Если существует такое число $M$, что для всякого $x \in B$ выполнены неравенства

$$
\left\|x_{+}\right\| \leqslant M\|x\|, \quad\left\|x_{-}\right\| \leqslant M\|x\|,
$$

то говорят, что конус обладает свойством несплющенности. Конус называют сильно миниэдральныц, если нижняя (верхняя) граница есть у каждого ограниченного по конусу множества.

Конус $\Lambda$ назьвается вполне правильньцм, если сходится каждая ограниченная по норме, невозрастающая (неубывающая) последовательность $x_{1} \triangleright x_{2} \triangleright \cdots \triangleright$ $x_{n} \cdots$. Если из $0 \triangleleft x \triangleleft y$ следует $\|x\| \leqslant m\|y\|$, где $m$ не зависит от $x$ и $y$, то конус $\Lambda$ называют нормальныцм. В этом случае, если $x \triangleleft y \triangleleft z$, то $\|y\| \leqslant(m+1) \times$ $(\|x\|+\|z\|)$, т.е. из ограниченности по конусу следует ограниченность по норме. Действительно, $0 \triangleleft y-x \triangleleft z-x$. Поэтому $\|y\|-\|x\| \leqslant m(\|z\|+\|x\|)$.

Покажем, что если конус $\Lambda \subset B$ нормальный миниэдральный и обладает свойством несплюшенности, то существует такое число $k$, что

$$
\|\sup \{x, y\}\| \leqslant k(\|x\|+\|y\|), \quad\|\inf \{x, y\}\| \leqslant k(\|x\|+\|y\|) .
$$

Воспользуемся неравенствами $-x_{-} \triangleleft x \triangleleft \sup \{x, y\} \triangleleft x_{+}+y_{+}$. Получим $0 \triangleleft$ $\sup \{x, y\}+x_{-} \triangleleft x_{+}+x_{-}+y_{+}$. Отсюда $\|\sup \{x, y\}\|-\left\|x_{-}\right\| \leqslant m\left(\left\|x_{+}\right\|+\right.$ $\left.\left\|x_{-}\right\|+\left\|y_{+}\right\|\right)$и $\|\sup \{x, y\}\| \leqslant m\left\|x_{+}\right\|+(m+1)\left\|x_{-}\right\|+m\left\|y_{+}\right\| \leqslant M(2 m+1)\|x\|+$ $M m\|y\| \leqslant M(2 m+1)(\|x\|+\|y\|)$. Второе неравенство доказывается аналогично.

Оператор $K: B \rightarrow B$ называют монотонныц, если из $x \triangleright y$ следует $K x \triangleright$ $K y$. Оператор $K: B \rightarrow B$ называется предельно монотонно компактным на ограниченном множестве $U \subset B$ [17; гл. $5, \S 38$, пा. 38.1, 38.2], если сходится каждая последовательность

$$
x_{0} \triangleright K x_{1} \triangleright K^{2} x_{2} \triangleright \cdots \triangleright K^{n} x_{n} \cdots,
$$


где $x_{n}, K^{n} x_{n} \in U, n=0,1,2, \ldots$. Назовем оператор предельно монотонно комnактным, если он обладает таким свойством на каждом ограниченном множестве из $B$. Понятие предельной монотонной компактности охватывает широкие классы нелинейных операторов. K ним относятся операторы, некоторая степень которых компактна, различные типы уплотняющих операторов. Любой оператор является предельно монотонно компактньм, если конус вполне правильный [17].

Мы будем использовать следуюшее утверждение о неподвижных точках монотонных операторов, полученное М.А. Красносельским и А.В. Соболевьм.

Теорема 1. Пусть монотонный оператор $K: B \rightarrow B$ преобразует в себя ограниченное замкнутое множество $U \subset B$, на котором он является предельно монотонно компактным. Пусть, далее, $u \triangleright K u$ для некоторого $u \in U$. Тогда оператор $K$ имеет на $U$ по крайней мере одну неподвижную точку $x$, удовлетворяющую условию $x \triangleleft и$.

Доказательство приведено в [17; гл. 5, п. 38.2]. Рассмотрим некоторые конструкции, используемые в этом доказательстве, необходимые нам в дальнейшем. Положим $U_{0}=\{x \in U \mid x \triangleright K x\}$. Это множество непусто, так как $u \triangleright K u$. Определим на $U_{0}$ при каждом натуральном $j$ функционал $\varphi_{j}$ равенством

$$
\varphi_{j} x=\sup \left\|K^{j} w-K^{j} y\right\|,
$$

где верхняя грань взята по всем таким $w, y \in U_{0}$, что $x \triangleright K^{j} w \triangleright K^{j} y$. Последовательность $\varphi_{j} x$ не возрастает, и поэтому $\varphi_{j}$ точечно сходится на $U_{0}$ к некоторому функционалу $\varphi$. Для этого предельного функционала выполнено $\varphi\left(x_{1}\right) \geqslant \varphi\left(x_{2}\right)$ при $x_{1} \triangleright x_{2}$.

Далее строится такая последовательность (3), что $x_{0}=u$ и $x_{n} \in U_{0}, \varphi\left(K^{n} x_{n}\right)<$ $1 / n$ при любом натуральном $n$. Эта последовательность сходится к некоторому элементу $z \in U$, являюшемуся неподвижной точкой оператора $K$.

ТЕОРема 2. Пусть выполнень условия теоремь 1. Пусть, кроме того, конус $\Lambda \subset B$ является миниэдральным $u \inf \{w, \vartheta\} \in U$ для любых двух неподвижных точек $w, \vartheta \in U$ оператора $K$. Тогда в множестве неподвижних точек оператора $K$ существует наименьиий элемент.

ДокАЗАТЕЛЬСтво. Покажем, что неподвижная точка $z$ оператора $K$, построенная при доказательстве предыдушего утверждения, является наименшшей. Напомним, что $u \triangleright K^{n} x_{n} \triangleright z$, где $\varphi\left(K^{n} x_{n}\right)<1 / n$ при любом $n=1,2, \ldots$. Предположим, что сушествует другая неподвижная точка $\bar{z}$ оператора $K$. Определим $\zeta=\inf \{z, \bar{z}\}$. Тогда $z \triangleright \zeta$, откуда $z=K z \triangleright K \zeta$. Аналогично, $\bar{z} \triangleright \zeta$ и $\bar{z}=K \bar{z} \triangleright K \zeta$. Следовательно, $\zeta \triangleright K \zeta, \zeta \in U_{0}$. Очевидно, при каждых $n, j$ вьполнены неравенства

$$
K^{n} x_{n} \triangleright K^{n+j} x_{n+j} \triangleright z \triangleright \zeta \triangleright K \zeta \triangleright \cdots \triangleright K^{n+j} \zeta .
$$

Существует такой номер $j(n)$, что $\varphi_{j(n)}\left(K^{n} x_{n}\right)<1 / n$. Из определения функционала $\varphi_{j(n)}$ следует $\left\|K^{n+j(n)} x_{n+j(n)}-K^{n+j(n)} \zeta\right\|<1 / n$. Переходя к пределу, получаем $\lim _{n \rightarrow \infty}\left\|K^{n+j(n)} x_{n+j(n)}-K^{n+j(n)} \zeta\right\|=0$. Отсюда и из неравенства $z-\zeta \triangleleft K^{n+j(n)} x_{n+j(n)}-K^{n+j(n)} \zeta$ имеем оценку $z \triangleleft \zeta$. Следовательно, $z=\zeta$. 
СЛЕДСТВИЕ 1. Пусть выполнены условия теоремы 1. Пусть, далее, конус $\Lambda \subset B$ является миниэдральным и для любых двух әлементов $x, y \in U$ существуют такие натуральные числа $n, j$, что $\inf \left\{K^{n} x, K^{j} y\right\} \in U$. Тогда в множсестве неподвижсных точек оператора $K$ существует наименьиий элемент.

Действительно, для неподвижных точек $w, \vartheta \in U$ оператора $K$ выполнены равенства $K^{n} w=w, K^{j} \vartheta=\vartheta$. Следовательно, $\inf \{w, \vartheta\} \in U$ и на основании теоремы 2 в множестве неподвижных точек оператора $K$ сушествует наименьший элемент.

СлЕДСТВИЕ 2. В условиях теоремы 2 в множестве неподвижных точек оператора $K$ существует наибольший элемент.

Для доказательства этого факта заметим, что так как $z=K z$, то $z \triangleleft K z$. Теперь осталось взять в качестве конуса в пространстве $B$ множество $-\Lambda$.

2. Приведенные вьше определения и утверждения нам придется несколько изменить, чтобы их “приспособить" к исследованию вольтерровых операторных неравенств.

Поставим в соответствие каждому $\gamma \in[0, b-a]$ некоторое измеримое множество $e_{\gamma}$ с мерой $\mu\left(e_{\gamma}\right)=\gamma$ таким образом, что

$$
\forall \gamma, \eta \in[0, b-a] \quad \gamma<\eta \Rightarrow e_{\gamma} \subset e_{\eta} .
$$

Обозначим совокупность этих множеств через $v$. Рассмотрим банахово пространство $B$ функций $f:[a, b] \rightarrow \mathbb{R}^{m}$. Будем говорить, что в пространстве $B$ выполнено $V$-условие, если для любого множества $e_{\gamma} \in v$ и для любой сходящейся последовательности $\left\{y_{i}\right\} \subset B,\left\|y_{i}-y\right\|_{B} \rightarrow 0$, из равенства $y_{i}(t)=0, i=1,2, \ldots$, при всех $t \in e_{\gamma}$ следует, что и предельная функция $y(t)=0$ при $t \in e_{\gamma}$.

Обозначим через $B\left(e_{\gamma}\right)$ пространство сужений функций из $B$ на множество $e_{\gamma}$. Норму в пространстве $B\left(e_{\gamma}\right)$ зададим формулой $\left\|y_{\gamma}\right\|_{B\left(e_{\gamma}\right)}=\inf \|y\|_{B}$, где нижняя грань берется по всевозможным продолжениям $y \in B$ функции $y_{\gamma} \in B\left(e_{\gamma}\right)$. Если вьполнено $V$-условие, то [18; гл. IV,$\S 1$, пा. $1.8,1.9]$ при таком определении нормы пространство $B\left(e_{\gamma}\right)$ является банаховым. Определим оператор $\Pi_{\gamma}: B \rightarrow B\left(e_{\gamma}\right)$ равенством $\left(\Pi_{\gamma} y\right)(t)=y(t)$ при всех $t \in e_{\gamma}$. Пусть оператор $P_{\gamma}: B\left(e_{\gamma}\right) \rightarrow B$ некоторым образом продолжает каждую функцию $y_{\gamma}$ на весь $[a, b]$. Зададим отображение $Z:(0, b-a] \times B \rightarrow \mathbb{R}$ формулой $Z(\gamma, y)=\left\|\Pi_{\gamma} y\right\|_{B\left(e_{\gamma}\right)}$. При каждом фиксированном $y \in B$ функция $Z(\cdot, y)$ не убывает и поэтому сушествует $\lim _{\gamma \rightarrow 0+0} Z(\gamma, y)=z_{0}(y)$. Доопределим отображение $Z$ значением $Z(0, y)=z_{0}(y)$.

Если $\Lambda$ - некоторьй конус пространства $B$, то множество $\Lambda_{\gamma}=\Pi_{\gamma} \Lambda$, состоящее из сужений на $e_{\gamma}$ функций, принадлежащих $\Lambda$, может и не являться конусом. Так, например, образуем в пространстве $L_{[0,3]}$ конус из функций $y:[0,3] \rightarrow \mathbb{R}$, удовлетворяющих условиям:

$$
\begin{array}{ll}
y(t) \geqslant 0 & \text { при почти всех } t \in[0,1], \\
y(t) \leqslant 0 & \text { при почти всех } t \in(1,2], \\
y(t)=\int_{0}^{2} y(s) d s & \text { при почти всех } t \in(2,3] .
\end{array}
$$

Сужениями этих функций на $[2,3]$ будут всевозможные константы, множество которых не является конусом. 
Будем говорить, что конус $\Lambda$ в пространстве $B$ является вольтерровым на $v$, если при любом $\gamma \in(0, b-a)$ множество $\Lambda_{\gamma}=\Pi_{\gamma} \Lambda$ будет конусом в пространстве $B\left(e_{\gamma}\right)$. Для элементов $x_{\gamma}, y_{\gamma} \in B\left(e_{\gamma}\right)$ таких, что $x_{\gamma}-y_{\gamma} \in \Lambda_{\gamma}$, сохраним обозначения $x_{\gamma} \triangleright y_{\gamma}, y_{\gamma} \triangleleft x_{\gamma}$. Важно заметить, что конус $\Lambda_{\gamma}$ не обязательно наследует свойства конуса $\Lambda$, о чем свидетельствуют следующие примеры.

ПримеР 1. В пространстве $L_{[0,2]}$ введем конус $\Lambda$ таких функций $x:[0,2] \rightarrow \mathbb{R}$, что $x(t) \geqslant 0$ при почти всех $t \in[0,1]$ и $x(t) \geqslant \int_{0}^{1} x(s) d s$ при почти всех $t \in(1,2]$. Точной верхней границей любых двух функций $x, y \in L_{[0,2]}$ является функция $u \in L_{[0,2]}$, определяемая формулой $u(t)=\sup \{x(t), y(t)\}$, если $t \in[0,1]$, и

$$
u(t)=\sup \left\{x(t)+\int_{0}^{1}(u(s)-x(s)) d s, y(t)+\int_{0}^{1}(u(s)-y(s)) d s\right\},
$$

если $t \in(1,2]$. Итак, конус $\Lambda$ миниэдральньй. Сужения принадлежащих $\Lambda$ функций на отрезок $[1,2]$ образуют множество $\Lambda_{1}$ неотрицательных функций, являющееся миниэдральньм конусом в $L_{[1,2]}$. Однако для двух функций $x, y \in L_{[0,2]}$ и их сужений $x_{1}, y_{1} \in L_{[1,2]}$ будет $\sup \{x, y\}(t) \neq \sup \left\{x_{1}, y_{1}\right\}(t), t \in(1,2]$, если только $x(t) \neq 0$ или $y(t) \neq 0$ на некотором подмножестве положительной меры отрезка $[0,1]$.

ПримеР 2. В пространстве $C_{[0,2]}$ рассмотрим конус $\Lambda$ функций $x:[0,2] \rightarrow \mathbb{R}$ таких, что $x(t) \geqslant 0$ при $t \in[0,1] ; x(0)=x(1)=0 ; x(t)=\varkappa \cdot(t-1)$ при $t \in(1,2]$, где $\varkappa \geqslant \max _{s \in[0,1]} x(s)$. Возьмем ограниченную возрастаюшую последовательность $\left\{x_{n}\right\} \subset C_{[0,2]}$. Тогда числовая последовательность $\left\{x_{n}(2)\right\}$ монотонно ограничена и, следовательно, сходится:

$$
\forall \varepsilon>0 \quad \exists J \quad \forall i, j>J \quad\left|x_{i}(2)-x_{j}(2)\right|<\varepsilon .
$$

Отсюда $\max _{s \in[0,1]}\left|x_{i}(s)-x_{j}(s)\right|<\varepsilon$. Кроме того, при всех $t \in[1,2]$ имеем

$$
\left|x_{i}(t)-x_{j}(t)\right|<\left|x_{i}(2)-x_{j}(2)\right|<\varepsilon .
$$

Итак, $\left\|x_{i}-x_{j}\right\|_{C}<\varepsilon$, т.е. последовательность $\left\{x_{n}\right\} \subset C_{[0,2]}$ фундаментальная. Тем самым доказано, что конус $\Lambda$ вполне правильньй.

Множество $\Lambda_{1}$, образованное сужениями функций из $\Lambda$ на $[0,1]$, состоит из всех неотрицательных функций, равных нулю на концах отрезка. Это множество - конус в пространстве $C_{[0,1]}$, не являющийся вполне правильным. Например, последовательность функций $x_{n}(t)=\left(t-t^{2}\right)^{1 / n}$ ограничена монотонна, но не является сходящейся в $C_{[0,1]}$.

ПримеР 3. В пространстве $C_{[-2,2]}$ определим конус $\Lambda$ функций $x:[-2,2] \rightarrow \mathbb{R}$, удовлетворяющих условиям:

$$
\begin{aligned}
& x(-1)=x(1)=0 ; \\
& x(t)=\underline{\varkappa}_{x} \cdot(t+1) \quad \text { при } t \in[-2,-1), \quad \text { где } \underline{\varkappa}_{x} \leqslant \min _{s \in[-1,0]} x(s) ; \\
& x(t)=\bar{\varkappa}_{x} \cdot(t-1) \quad \text { при } t \in(1,2], \quad \text { где } \bar{\varkappa}_{x} \geqslant \max _{s \in[0,1]} x(s) .
\end{aligned}
$$

Пусть $y \triangleright x \triangleright 0$. Тогда

$$
\max _{s \in[0,2]} y(s)=y(2)=\bar{\varkappa}_{y} \geqslant \max _{s \in[0,2]} x(s)=x(2)=\bar{\varkappa}_{x} \geqslant 0 .
$$


Обозначим $z=y-x$. Имеем $z \triangleright 0, \bar{\varkappa}_{z}=\bar{\varkappa}_{y}-\bar{\varkappa}_{x}$. Далее,

$$
\begin{aligned}
\bar{\varkappa}_{z} & \geqslant \max _{s \in[0,1]}(y(s)-x(s)) \geqslant \min _{s \in[0,1]} y(s)+\max _{s \in[0,1]}(-x(s)) \\
& =\left|\min _{s \in[0,1]} x(s)\right|-\left|\min _{s \in[0,1]} y(s)\right|=\left|\min _{s \in[0,2]} x(s)\right|-\left|\min _{s \in[0,2]} y(s)\right| .
\end{aligned}
$$

Следовательно,

$$
\left|\min _{s \in[0,2]} x(s)\right| \leqslant \bar{\varkappa}_{y}+\left|\min _{s \in[0,2]} y(s)\right| \leqslant 2 \max _{s \in[0,2]}|y(s)| .
$$

Отсюда и из (5) получаем $\max _{s \in[0,2]}|x(s)| \leqslant 2 \max _{s \in[0,2]}|y(s)|$. Аналогично доказывается, что $\max _{s \in[-2,0]}|x(s)| \leqslant 2 \max _{s \in[-2,0]}|y(s)|$. Таким образом, $\|x\|_{C} \leqslant$ $2\|y\|_{C}$, т.е. конус $\Lambda$ нормальньй.

Образуем множество $\Lambda_{1}$ сужений принадлежащих $\Lambda$ функций на $[-1,2]$, являюшееся конусом в пространстве $C_{[-1,2]}$. Для функций $y, x_{n} \in C_{[-1,2]}$,

$$
y(t)=\left\{\begin{array}{ll}
0, & \text { если } t \in[-1,1], \\
2(t-1), & \text { если } t \in(1,2],
\end{array} \quad x_{n}(t)= \begin{cases}n t, & \text { если } t \in[-1,0], \\
0, & \text { если } t \in[0,1], \\
t-1, & \text { если } t \in(1,2],\end{cases}\right.
$$

имеем $y \triangleright x_{n} \triangleright 0$ и $\|y\|=2,\left\|x_{n}\right\|=n$. Так как можно выбирать значения $n$ сколь угодно большими, то конус $\Lambda_{1}$ не является нормальным.

Вольтерров конус $\Lambda \subset B$ назовем вольтеррово миниәдральным, если при любом $\gamma \in(0, b-a]$ конус $\Lambda_{\gamma}=\Pi_{\gamma} \Lambda$ в пространстве $B\left(e_{\gamma}\right)$ является миниэдральньм, причем для любых двух элементов $x, y \in B$ выполнено $\inf \left\{\Pi_{\gamma} x, \Pi_{\gamma} y\right\}=$ $\Pi_{\gamma} \inf \{x, y\}$.

ТЕОРемА 3. Пусть вольтерров конус $\Lambda \subset B$ является несплющенным $и$ вольтеррово миниәдральным. Тогда при любом $\gamma \in(0, b-a]$ конус $\Lambda_{\gamma} \subset B\left(e_{\gamma}\right)$ является также несплющенныц, причем если число $M$ удовлетворяет неравенствам (1), то при любом $\gamma \in(0, b-a]$ и всех $x_{\gamma} \in B\left(e_{\gamma}\right)$ выполнены неравенства

$$
\left\|x_{\gamma+}\right\| \leqslant M\left\|x_{\gamma}\right\|, \quad\left\|x_{\gamma-}\right\| \leqslant M\left\|x_{\gamma}\right\|,
$$

əде $x_{\gamma+}=\sup \left\{x_{\gamma}, 0\right\}, x_{\gamma-}=\sup \left\{-x_{\gamma}, 0\right\}$.

ДокАЗАТЕЛЬСТво. Возьмем любоеположительное число $\varepsilon$. Продолжим функцию $x_{\gamma} \in B\left(e_{\gamma}\right), x_{\gamma} \neq 0$, до функции $x \in B$ так, чтобы $\left\|x_{\gamma}\right\| \geqslant\|x\|-\varepsilon$. Найдем $x_{+}=\sup \{x, 0\}$. В силу вольтерровой миниэдральности конуса $\Lambda$ функция $x_{+}$является продолжением функции $x_{\gamma+}$. Поэтому $\left\|x_{\gamma+}\right\| \leqslant\left\|x_{+}\right\| \leqslant M\|x\| \leqslant$ $M\left(\left\|x_{\gamma}\right\|+\varepsilon\right)$. Отсюда вследствие произвольности $\varepsilon$ получаем утверждение теоремы.

Вольтерров конус $\Lambda \subset B$ назовем вольтеррово сильно миниэдральныц, если для любого ограниченного по конусу множества $\Omega$ и для любого $\gamma \in(0, b-a]$ сушествует точная нижняя (верхняя) граница множества $\Omega_{\gamma}=\Pi_{\gamma} \Omega$, причем inf $\Omega_{\gamma}=$ $\Pi_{\gamma} \inf \Omega$. Вольтерров конус $\Lambda \subset B$ назовем вольтеррово вполне правильныцм, если при любом $\gamma \in(0, b-a]$ в пространстве $B\left(e_{\gamma}\right)$ сходится каждая ограниченная по норме невозрастающая (неубывающая) последовательность $x_{1 \gamma} \triangleright x_{2 \gamma} \triangleright$ $\cdots \triangleright x_{n \gamma} \cdots$. Если сушествует такая константа $m$, что при любом $\gamma \in(0, b-a]$ и всех $x_{\gamma}, y_{\gamma} \in B\left(e_{\gamma}\right)$ из $0 \triangleleft x_{\gamma} \triangleleft y_{\gamma}$ следует $\left\|x_{\gamma}\right\| \leqslant m\left\|y_{\gamma}\right\|$, то конус $\Lambda$ назовем вольтеррово нормальным. 
Если конус $\Lambda$ вольтерров на совокупности $v=\left\{e_{\gamma}\right\}$ и вольтеррово сильно миниэдральньй, то можно определить точные верхнюю и нижнюю границы функций, заданных на разных множествах. Пусть $x_{\tau} \in B\left(e_{\tau}\right), y_{\gamma} \in B\left(e_{\gamma}\right), 0<\tau<\gamma \leqslant b-a$. Обозначим через $x_{\gamma}$ некоторое продолжение функции $x_{\tau}$, принадлежашее пространству $B\left(e_{\gamma}\right)$. Верхней гранью функций $x_{\tau}, y_{\gamma}$ назовем элемент

$$
\sup \left\{x_{\tau}, y_{\gamma}\right\}=\inf _{x_{\gamma} \in B\left(e_{\gamma}\right)} \sup \left\{x_{\gamma}, y_{\gamma}\right\} \in B\left(e_{\gamma}\right) .
$$

Здесь infimum вычисляется по всевозможным продолжениям $x_{\gamma} \in B\left(e_{\gamma}\right)$ функции $x_{\tau}$. Так как для любого $x_{\gamma}$ имеет место $\sup \left\{x_{\gamma}, y_{\gamma}\right\} \triangleright y_{\gamma}$, то вследствие сильной миниэдральности конуса $\Lambda_{\gamma}$ заданная равенством (6) верхняя граница сушествует. Аналогично определим

$$
\inf \left\{x_{\tau}, y_{\gamma}\right\}=\sup _{x_{\gamma} \in B\left(e_{\gamma}\right)} \inf \left\{x_{\gamma}, y_{\gamma}\right\} \in B\left(e_{\gamma}\right)
$$

Заданные таким образом верхняя, нижняя грани обладают "привычными” свойствами:

1. $\sup \left\{x_{\tau}, y_{\gamma}\right\} \triangleright y_{\gamma}, \Pi_{\tau} P_{\gamma} \sup \left\{x_{\tau}, y_{\gamma}\right\} \triangleright x_{\tau} ;$

2. $\forall w_{\gamma} \in B\left(e_{\gamma}\right) w_{\gamma} \triangleright y_{\gamma}, \Pi_{\tau} P_{\gamma} w_{\gamma} \triangleright x_{\tau} \Longrightarrow w_{\gamma} \triangleright \sup \left\{x_{\tau}, y_{\gamma}\right\}$;

3. $x_{\tau} \triangleright \Pi_{\tau} P_{\gamma} y_{\gamma} \Longrightarrow \Pi_{\tau} P_{\gamma} \sup \left\{x_{\tau}, y_{\gamma}\right\}=x_{\tau}$.

Покажем, что если вольтерров на $v$ конус $\Lambda$ вольтеррово нормальньй, вольтеррово сильно миниэдральный и несплюшенный, то сушествует такое число $\widetilde{k}$, что при любых $\tau, \gamma, 0<\tau<\gamma \leqslant b-a, x_{\tau} \in B\left(e_{\tau}\right), y_{\gamma} \in B\left(e_{\gamma}\right)$, вьполнено

$$
\left\|\sup \left\{x_{\tau}, y_{\gamma}\right\}\right\| \leqslant \widetilde{k}\left(\left\|x_{\tau}\right\|+\left\|y_{\gamma}\right\|\right), \quad \inf \left\{x_{\tau}, y_{\gamma}\right\} \leqslant \widetilde{k}\left(\left\|x_{\tau}\right\|+\left\|y_{\gamma}\right\|\right) .
$$

Пусть $\varepsilon>0, \widetilde{x}_{\gamma}$ - такое продолжение функции $x_{\tau}$ на $e_{\gamma}$, что $\left\|\widetilde{x}_{\gamma}\right\| \leqslant\left\|x_{\tau}\right\|+\varepsilon$. Имеем неравенства

$$
y_{\gamma} \triangleleft \sup \left\{x_{\tau}, y_{\gamma}\right\}=\inf _{x_{\gamma} \in B\left(e_{\gamma}\right)} \sup \left\{x_{\gamma}, y_{\gamma}\right\} \triangleleft \sup \left\{\widetilde{x}_{\gamma}, y_{\gamma}\right\} .
$$

Теорема 3 позволяет воспользоваться формулой (2) для элементов пространства $B\left(e_{\gamma}\right)$ при любом $\gamma$. Таким образом,

$$
\left\|\sup \left\{x_{\tau}, y_{\gamma}\right\}\right\|-\left\|y_{\gamma}\right\| \leqslant k\left(\left\|\widetilde{x}_{\gamma}\right\|+\left\|y_{\gamma}\right\|\right)+\left\|y_{\gamma}\right\| .
$$

Отсюда $\left\|\sup \left\{x_{\tau}, y_{\gamma}\right\}\right\| \leqslant(k+2)\left(\left\|x_{\tau}\right\|+\varepsilon+\left\|y_{\gamma}\right\|\right)$. Из произвольности $\varepsilon$ следует справедливость формулы (8) с коэффициентом $\widetilde{k}=k+2$.

Необходимо отметить, что наименьшее из чисел $\widetilde{k}$, отвечающих условию (8), удовлетворяет неравенству $\widetilde{k} \geqslant 1$. Для доказательства этой оценки достаточно воспользоваться формулой (8) для элементов $x_{\tau} \triangleright 0, y_{\gamma}=0$.

3. Здесь будет получено утверждение об операторном неравенстве - основной результат этой работы.

Сформулируем определение, несколько расширяющее классическое определение вольтерровости, данное А.Н. Тихоновым [19]. Отображение $K: B \rightarrow B$ будем называть вольтерровым на системе $v$, если для каждого множества $e_{\gamma} \in v$ и любых функций $y, z \in B$ из $y(s)=z(s)$ на $e_{\gamma}$ следует $(K y)(s)=(K z)(s)$ на $e_{\gamma}$. 
Отметим, что обобшения понятия вольтерровости возникают в ряде теоретических и прикладных задач и привлекают многочисленных исследователей [20]-[29]. Приведенное нами определение не претендует на максимальную общность, но оказывается востребованным в теории функционально-дифференциальных уравнений $[24]-[26]$.

Рассмотрим уравнение

$$
x(t)-(K x)(t)=0, \quad t \in[a, b],
$$

с вольтерровым оператором $K: B \rightarrow B$. Если существует число $\gamma \in(0, b-a)$ и элемент $z_{\gamma} \in B\left(e_{\gamma}\right)$, удовлетворяющий равенству

$$
z_{\gamma}=\Pi_{\gamma} K P_{\gamma} z_{\gamma}
$$

то уравнение (9) будем назьвать локально разрешимым, а функцию $z_{\gamma}-$ локальным решением, определенным на $e_{\gamma}$. Элемент $z_{b-a} \in B$, удовлетворяющий уравнению (9), назовем глобальным решением. Будем говорить, что функция $z_{\eta}: \bigcup_{\gamma<\eta} e_{\gamma} \rightarrow \mathbb{R}^{m}, \eta \in(0, b-a]$, является предельно продолженным решением уравнения $(9)$, если при любом $\gamma \in(0, \eta)$ сужение $z_{\gamma}$ функции $z_{\eta}$ на множество $e_{\gamma}$ является локальным решением и $\lim _{\gamma \rightarrow \eta}\left\|z_{\gamma}\right\|=\infty$. Вследствие вольтерровости оператора $K$ сужение $z_{\gamma}$ решения $z_{\zeta}$ (локального, глобального или предельно продолженного) на произвольное множество $e_{\gamma}, \gamma \in(0, \zeta)$, будет локальным решением уравнения (9). Будем называть решение $z_{\gamma}$ частью решения $z_{\zeta}$, а решение $z_{\zeta}$ - продолжением решения $z_{\gamma}$.

Пусть пространство $B$ полуупорядочено вольтерровьм конусом $\Lambda$, вольтерров оператор $K: B \rightarrow B$ является монотонньп. Прежде всего заметим, что при любом $\gamma \in(0, b-a]$ оператор $K_{\gamma}=\Pi_{\gamma} K P_{\gamma}: B\left(e_{\gamma}\right) \rightarrow B\left(e_{\gamma}\right)$ также будет монотонным. Далее, отметим, что из предельной монотонной компактности оператора $K$ не следует наличие этого свойства у оператора $K_{\gamma}$. Действительно, упорядочим пространство $C_{[0,2]}$ конусом $\Lambda$, рассмотренньм в примере 2 . Так как этот конус вполне правильный, то тождественный оператор $I: C_{[0,2]} \rightarrow C_{[0,2]}$ является предельно монотонно компактным. Однако тождественный оператор $I_{1}=\Pi_{1} I P_{1}: C_{[0,1]} \rightarrow$ $C_{[0,1]}$ уже не является предельно монотонно компактньм, так как конус $\Lambda_{1}=\Pi_{1} \Lambda$ не будет вполне правильным. Назовем вольтерров оператор $K: B \rightarrow B$ вольтеррово предельно монотонно компактным, если для любого $\gamma \in(0, b-a]$ сходится каждая последовательность $x_{0 \gamma} \triangleright K_{\gamma} x_{1 \gamma} \triangleright K_{\gamma}^{2} x_{2 \gamma} \triangleright \cdots \triangleright K_{\gamma}^{n} x_{n \gamma} \cdots$, где $x_{n \gamma} \in B\left(e_{\gamma}\right), n=0,1,2, \ldots$

ТЕОРема 4. Пусть в банаховом пространстве $B$, удовлетворяющем $V$-условию, введено отношение порядка, порожсденое вольтерровым на системе $v$ конусом. Пусть оператор $K: B \rightarrow B$ является вольтерровым на $v$, монотонным, вольтеррово предельно монотонно компактным и удовлетворяет следующему условию:

$$
\begin{gathered}
\exists x_{0} \in B, \quad \exists r_{1}, \quad \exists r_{2}, \quad r_{1}>r_{2}>0, \quad \exists \delta>0 \quad \forall x \in B \\
\left\|x-x_{0}\right\| \leqslant r_{1} \Longrightarrow Z\left(\delta, K x-x_{0}\right) \leqslant r_{2} .
\end{gathered}
$$

Пусть, кроме того, для некоторого элемента $u \in B,\left\|u-x_{0}\right\| \leqslant r_{1}$, вьполнено неравенство $и \triangleright K u$. Тогда существует определенное на $e_{\delta}$ локальное решение $z_{\delta}$ уравнения (9), для которого имеет место оценка $z_{\delta} \triangleleft \Pi_{\delta} u$. 
Для доказательства достаточно проверить условия теоремы 1, взяв в пространстве $B\left(e_{\delta}\right)$ в качестве множества $U$ шар радиуса $r_{2}$ с центром в элементе $x_{0 \delta}=\Pi_{\delta} x_{0}$.

Чтобы сформулировать утверждение о продолжаемости решений уравнения (9), нам потребуется следующее определение. Оператор $K: B \rightarrow B$ назовем улучшающим на системе $v$, если

$$
\begin{gathered}
\exists \mu \quad \forall x \in B \quad Z(0, K x) \leqslant \mu, \\
\forall r>0, \quad \forall \varepsilon>0 \quad \exists \delta>0 \quad \forall x \in B, \quad \forall \tau, \gamma \in[0, b-a] \\
|\tau-\gamma|<\delta, \quad\|x\| \leqslant r \Longrightarrow|Z(\gamma, K x)-Z(\tau, K x)|<\varepsilon .
\end{gathered}
$$

Отметим, что даже после замены условия (10) более жестким требованием улучшаемости оператора $K: B \rightarrow B$ уравнение (9) может иметь непродолжаемые решения. В качестве примера такой ситуации рассмотрим уравнение, в котором оператор $K: C_{[0,1]} \rightarrow C_{[0,1]}$ определен следующим образом. Возьмем любую непрерывную функцию $x:[0,1] \rightarrow \mathbb{R}$ и рассмотрим уравнение

$$
\int_{0}^{\xi}\left(\operatorname{arctg} x(s)-\frac{\pi}{2}\right) d s=-1
$$

относительно неизвестного $\xi \in[0,1]$. Функция $\Theta(\cdot)=\int_{0}^{(\cdot)}\left(\operatorname{arctg} x(s)-\frac{\pi}{2}\right) d s$ убывает и $\Theta(0)=0$; поэтому если решение уравнения (13) сушествует, то оно единственно. В случае несовместности уравнения (13) полагаем

$$
(K x)(t)=\frac{1}{2 \sqrt{2}} \int_{0}^{t} \sqrt{|x(s)-x(0)|+x(s)-x(0)} d s \text { при всех } t \in[0,1] .
$$

Если уравнение (13) разрешимо, то

$$
\begin{aligned}
& (K x)(t)=\frac{1}{2 \sqrt{2}} \int_{0}^{t} \sqrt{|x(s)-x(0)|+x(s)-x(0)} d s \quad \text { при } t \in[0, \xi], \\
& (K x)(t)=(K x)(\xi)-\int_{\xi}^{t}(0.5+\operatorname{sign}(x(s)-x(0))) d s \quad \text { при } t \in(\xi, b] .
\end{aligned}
$$

Определенный таким образом оператор $K$ действует в пространстве непрерывных на $[0,1]$ функций, является вольтерровым по А. Н. Тихонову и улучшающим. Упорядочим пространство $C_{[0,1]}$ конусом $\Lambda$, содержашим неотрицательные функции-константы. Этот конус вольтерров на системе $\{[0, \gamma]\}$ и вольтеррово вполне правильный. Если $x_{1}(t)-x_{2}(t)=$ const $=c \geqslant 0$, то при всех $t$ выполнено $x_{1}(t)-x_{1}(0)=x_{2}(t)-x_{2}(0)$ и поэтому $\left(K x_{1}\right)(t)-\left(K x_{2}\right)(t) \equiv 0$. Следовательно, оператор $K$ монотонен. Так как конус $\Lambda$ вольтеррово вполне правильный, то оператор $K$ вольтеррово монотонно компактный. В завершение проверки условий теоремы 4 заметим, что функция

$$
u(t)= \begin{cases}1+t^{2}, & \text { если } t \in[0 ; 0.72], \\ 1.52-1.5(t-0.72), & \text { если } t \in(0.72 ; 1],\end{cases}
$$

удовлетворяет равенству $(K u)(t)=u(t)-1$, т.е. $u \triangleright K u$. 


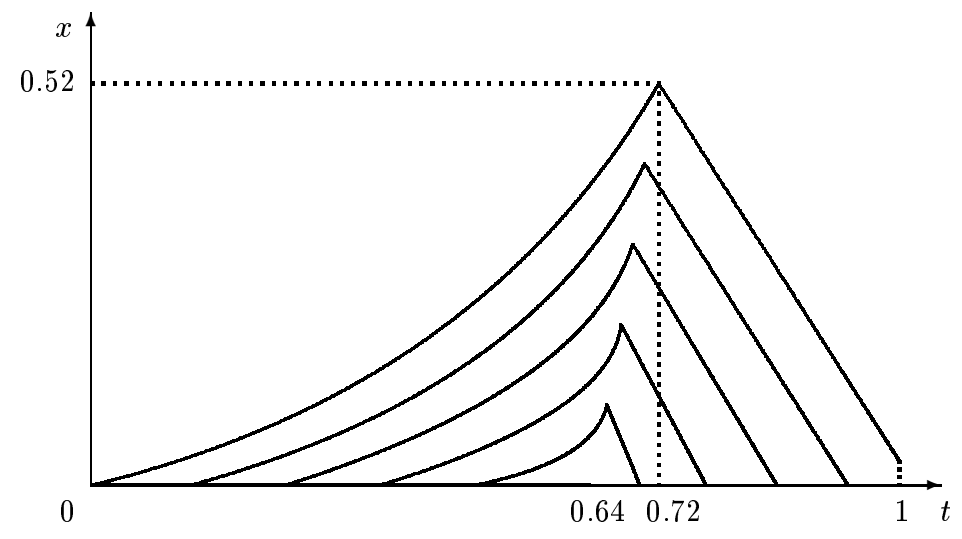

Рис. 1. Множество решений

Множество решений рассматриваемого уравнения представлено на рис. 1.

Уравнение имеет непродолжаемые решения. Например, решение $x(t) \equiv 0$, $t \in[0 ; 0.64]$, "умирает" в точке $t=0.64$. Отметим также, что если рассмотреть сужения всех решений на любой отрезок $[0, \delta], \delta \leqslant 0.64$, где все они определены, то окажется, что любые два решения $x_{1}, x_{2}$ не упорядочены, т.е. $x_{1} \not x_{2}$ и $x_{1} \nless x_{2}$. Как показывают приводимые ниже утверждения, продолжаемость решений уравнения (9) и сушествование в множестве решений наименњшего элемента гарантируются геометрическими свойствами конуса $\Lambda$.

ТЕОРема 5. Пусть в банаховом пространстве $B$, удовлетворяющем $V$-условию, введено отношение порядка, порожденное несплющенным, вольтерровым на системе $v$, вольтеррово сильно миниэдральным и вольтеррово нормальным конусом $\Lambda$. Пусть оператор $K: B \rightarrow B$ является улучшающим, вольтерровым на $v$, монотонным и вольтеррово предельно монотонно компактным. Пусть, кроме того, для некоторого әлемента $u \in B$ выполнено неравенство $и \triangleright K u$. Тогда любое локальное решение $z_{\gamma}$ уравнения (9), для которого имеет место оченка $z_{\gamma} \triangleleft \Pi_{\gamma}$, продолжсаемо либо до глобального $z_{b-a}$, удовлетворяющего неравенству $z_{b-a} \triangleleft и$, либо до предельно продолжсенного решения $z_{\eta}$ такого, что при всех $\delta<\eta$ выполнено $z_{\delta} \triangleleft \Pi_{\delta} u$.

ДоКАЗАТЕльство. Возьмем любое локальное решение $z_{\gamma}$, определенное на $e_{\gamma}$ и удовлетворяющее неравенству $z_{\gamma} \triangleleft \Pi_{\gamma} u$. Найдем $\varphi_{1}=\inf \left\{z_{\gamma}, u\right\}$. Так как $u \triangleright K u$, то $u \triangleright K \varphi_{1}$; и так как $z_{\gamma}=K_{\gamma} z_{\gamma}$, то $z_{\gamma} \triangleright \Pi_{\gamma} K \varphi_{1}$. Следовательно, $\varphi_{1} \triangleright K \varphi_{1}$. Кроме того, $\varphi_{1}(t)=z_{\gamma}(t)$ при $t \in e_{\gamma}$.

Выберем $r=\left\|\varphi_{1}\right\|+1, \varepsilon=1 / 2$ и найдем $\delta_{1}$, являющееся точной верхней границей всевозможных чисел $\delta$, удовлетворяющих требованию (12) в условии улучшаемости оператора $K$. Обозначим $\gamma_{1}=\gamma+\delta_{1} / 2$. Для любого такого $x \in B$, что $\|x\|<\left\|z_{\gamma_{1}}\right\|+1, x(t)=z_{\gamma}(t)$ при $t \in e_{\gamma}$, выполнено

$$
Z\left(\gamma_{1}, K x\right)=\left(Z\left(\gamma_{1}, K x\right)-Z(\gamma, K x)\right)+Z(\gamma, K x)<\frac{1}{2}+\left\|\varphi_{1}\right\| .
$$

В пространстве $B\left(e_{\gamma_{1}}\right)$ выделим замкнутый шар радиуса $\left\|\varphi_{1}\right\|+1$, а в нем - множество $U_{1}$ функций, совпадающих при $t \in e_{\gamma} \mathrm{c} z_{\gamma}(t)$ (или $\varphi_{1}(t)$, что одно и то же). 
Множество $U_{1}$ замкнуто. Продолжим оператором $P_{\gamma_{1}}$ каждую функцию из $U_{1}$ так, чтобы ее норма увеличилась менее чем на $1 / 2$. Для таких продолжений можем воспользоваться неравенством (14), из которого будет следовать включение $\Pi_{\gamma_{1}} K P_{\gamma_{1}} U_{1} \subset U_{1}$. Так как оператор $K_{\gamma_{1}}=\Pi_{\gamma_{1}} K P_{\gamma_{1}}: B\left(e_{\gamma_{1}}\right) \rightarrow B\left(e_{\gamma_{1}}\right)$ монотонный и предельно монотонно компактный, а элемент $\varphi_{\gamma_{1}}=\Pi_{\gamma_{1}} \varphi_{1} \in U_{1}$ удовлетворяет неравенству $\varphi_{\gamma_{1}} \triangleright K_{\gamma_{1}} \varphi_{\gamma_{1}}$, то согласно теореме 1 оператор $K_{\gamma_{1}}$ имеет неподвижную точку $z_{\gamma_{1}}$. Это локальное решение уравнения (9), определенное на $e_{\gamma_{1}}$, являющееся продолжением решения $z_{\gamma}$ и удовлетворяющее неравенству $z_{\gamma_{1}} \triangleleft P_{\gamma_{1}} u$.

Найдем $\varphi_{2}=\inf \left\{z_{\gamma_{1}}, u\right\}$. Имеем $\varphi_{2} \triangleright K \varphi_{2} ; \varphi_{2}(t)=\varphi_{1}(t)$ при $t \in e_{\gamma_{1}}$; $\varphi_{2}(t)=z_{\gamma}(t)$ при $t \in e_{\gamma}$. Выберем $r=\left\|\varphi_{2}\right\|+1, \varepsilon=1 / 2$ и найдем $\delta_{2}$, являющееся точной верхней границей всевозможных чисел $\delta$, удовлетворяющих условию (12). Обозначим $\gamma_{2}=\gamma_{1}+\delta_{2} / 2$. Замкнутое множество $U_{2}=\left\{x_{\gamma_{2}} \in B\left(e_{\gamma_{2}}\right) \mid\right.$ $\left.\left\|x_{\gamma_{2}}\right\| \leqslant\left\|\varphi_{2}\right\|+1 \wedge x_{\gamma_{2}}(t)=\varphi_{2}(t), t \in e_{\gamma_{1}}\right\}$ инвариантно относительно монотонного и предельно монотонно компактного оператора $K_{\gamma_{2}}=\Pi_{\gamma_{2}} K P_{\gamma_{2}}: B\left(e_{\gamma_{2}}\right) \rightarrow$ $B\left(e_{\gamma_{2}}\right)$. Элемент $\varphi_{\gamma_{2}}=\Pi_{\gamma_{2}} \varphi_{2} \in U_{2}$ удовлетворяет неравенству $\varphi_{\gamma_{2}} \triangleright K_{\gamma_{2}} \varphi_{\gamma_{2}}$. Таким образом, существует локальное решение $z_{\gamma_{2}}$ уравнения (9), определенное на $e_{\gamma_{2}}$, являюшееся продолжением решения $z_{\gamma_{1}}$ и удовлетворяюшее неравенству $z_{\gamma_{2}} \triangleleft P_{\gamma_{2}} u$.

Докажем, что, продолжая описанные построения, мы получим глобальное или предельно продолженное решение. Вычислим $\lim _{i \rightarrow \infty} \gamma_{i}=\eta$ и рассмотрим функцию $z_{\eta}$, определенную на $\bigcup_{i=1}^{\infty} e_{\gamma_{i}}$, сужением которой на $e_{\gamma_{i}}$ будет $z_{\gamma_{i}}, i=1,2, \ldots$ Если функция $z_{\eta}$ не является предельно продолженным решением, то найдется такое число $N$, что при всех натуральных $i$ имеет место $\left\|z_{\gamma_{i}}\right\| \leqslant N$. Согласно формуле (8) получаем $\left\|\varphi_{i}\right\| \leqslant \widetilde{k}(N+\|u\|)$. Возьмем $r=\widetilde{k}(N+\|u\|)+1, \varepsilon=1 / 2$ и найдем $\bar{\delta}$, являющееся точной верхней границей всевозможных чисел $\delta$, удовлетворяюших условию (12). Таким образом, при любом $i$ выполнено $\delta_{i} \geqslant \bar{\delta}$. Это означает, что за конечное число шагов будет построено глобальное решение уравнения (9).

Теорема доказана.

Глобальное решение $z_{b-a}$ уравнения (9) с вольтерровым на $v$ оператором $K: B \rightarrow B$ назовем нижним (верхним), если для любого локального решения $y_{\gamma} \in B\left(e_{\gamma}\right)$ выполнено $y_{\gamma} \triangleright z_{\gamma}=\Pi_{\gamma} z_{b-a} \quad\left(y_{\gamma} \triangleleft z_{\gamma}=\Pi_{\gamma} z_{b-a}\right)$ и для всякого глобального решения $y_{b-a}$ также вьполнено $y_{b-a} \triangleright z_{b-a}\left(y_{b-a} \triangleleft z_{b-a}\right)$. Предельно продолженное решение $z_{\eta}$ назовем нижним (верхним), если для каждого локального решения $y_{\gamma}, \gamma<\eta$, имеет место неравенство $y_{\gamma} \triangleright z_{\gamma}$ $\left(y_{\gamma} \triangleleft z_{\gamma}\right)$, где $z_{\gamma}-$ сужение на $e_{\gamma}$ функции $z_{\eta}$.

Теорема 6. При выполнении условий теоремы 5 существует либо нижнее глобальное решение $z_{b-a}$ уравнения (9) такое, что $z_{b-a} \triangleleft и$, либо нижнее предельно продолэсенное решение $z_{\eta}$, сужение которого $z_{\gamma}$ на любое $e_{\gamma}$, $\gamma<\eta$, удовлетворяет неравенству $z_{\gamma} \triangleleft \Pi_{\gamma} u$.

ДоКАЗАТЕЛЬСТво. Пусть $\mu$-константа, сушествование которой гарантируется свойством (11). Возьмем $\varepsilon=1, r=\widetilde{k}(\|u\|+2 \mu+3)+1$, где $\widetilde{k} \geqslant 1$ удовлетворяет неравенству (8). Найдем точную верхнюю границу $\delta_{1}$ всевозможных чисел $\delta$, отвечающих условию (12). Обозначим $\gamma_{1}=\delta_{1} / 2$. Для любого $x \in B$, 


$$
\begin{aligned}
\|x\|<\widetilde{k}(\|u\|+2 \mu+3) & +1, \text { вьполнено } \\
Z\left(\gamma_{1}, K x\right) & =\left(Z\left(\gamma_{1}, K x\right)-Z(0, K x)\right)+Z(0, K x)<1+\mu .
\end{aligned}
$$

В пространстве $B\left(e_{\gamma_{1}}\right)$ выделим замкнутый шар $U_{1}$ радиуса $\widetilde{k}(\|u\|+2 \mu+3)$. Оператором $P_{\gamma_{1}}$ продолжим каждую функцию из $B\left(e_{\gamma_{1}}\right)$ на весь отрезок $[a, b]$ так, чтобы ее норма увеличилась менее чем на 1 . Для полученных таким образом функций можем воспользоваться неравенством (15), из которого получаем включение $\Pi_{\gamma_{1}} K P_{\gamma_{1}} U_{1} \subset U_{1}$. Так как оператор $K_{\gamma_{1}}=\Pi_{\gamma_{1}} K P_{\gamma_{1}}: B\left(e_{\gamma_{1}}\right) \rightarrow B\left(e_{\gamma_{1}}\right)$ монотонный и предельно монотонно компактньй, а элемент $u_{\gamma_{1}}=\Pi_{\gamma_{1}} u \in U_{1}$ удовлетворяет неравенству $u_{\gamma_{1}} \triangleright K_{\gamma_{1}} u_{\gamma_{1}}$, то согласно теореме 2 среди локальных решений, принадлежаших множеству $U_{1}$, есть наименьшее решение $z_{\gamma_{1}}$. Это решение удовлетворяет неравенству $z_{\gamma_{1}} \triangleleft P_{\gamma_{1}} u$.

Найдем $\varphi_{1}=\inf \left\{z_{\gamma_{1}}, u\right\}$. Имеем $\varphi_{1} \triangleright K \varphi_{1} ; \varphi_{1}(t)=z_{\gamma_{1}}(t)$ при $t \in e_{\gamma_{1}}$. Выберем $r=\widetilde{k}\left(\left\|\varphi_{1}\right\|+2\left\|z_{\gamma_{1}}\right\|+3\right)+1, \varepsilon=1$ и найдем $\delta_{2}$, являюшееся точной верхней границей всевозможных чисел $\delta$, удовлетворяющих условию (12). Обозначим $\gamma_{2}=\gamma_{1}+\delta_{2} / 2$. Для любого $x \in B$ такого, что $\|x\| \leqslant \widetilde{k}\left(\left\|\varphi_{1}\right\|+2\left\|z_{\gamma_{1}}\right\|+3\right)+1$ и $x(t)=z_{\gamma_{1}}(t)$ при $t \in e_{\gamma_{1}}$, выполнено

$$
Z\left(\gamma_{2}, K x\right)=\left(Z\left(\gamma_{2}, K x\right)-Z\left(\gamma_{1}, K x\right)\right)+Z\left(\gamma_{1}, K x\right)<\left\|z_{\gamma_{1}}\right\|+1 .
$$

Из этого неравенства следует инвариантность замкнутого множества

$$
U_{2}=\left\{x_{\gamma_{2}} \in B\left(e_{\gamma_{2}}\right) \mid\left\|x_{\gamma_{2}}\right\| \leqslant \widetilde{k}\left(\left\|\varphi_{1}\right\|+2\left\|z_{\gamma_{1}}\right\|+3\right) \wedge x_{\gamma_{2}}(t)=\varphi_{1}(t), t \in e_{\gamma_{1}}\right\}
$$

относительно монотонного и предельно монотонно компактного оператора $K_{\gamma_{2}}=$ $\Pi_{\gamma_{2}} K P_{\gamma_{2}}: B\left(e_{\gamma_{2}}\right) \rightarrow B\left(e_{\gamma_{2}}\right)$. Элемент $\varphi_{\gamma_{2}}=\Pi_{\gamma_{2}} \varphi_{1} \in U_{2}$ удовлетворяет неравенству $\varphi_{\gamma_{2}} \triangleright K_{\gamma_{2}} \varphi_{\gamma_{2}}$. Таким образом, существует локальное решение $z_{\gamma_{2}}$ уравнения (9), определенное на $e_{\gamma_{2}}$, являюшееся наименьшим из решений, принадлежаших множеству $U_{2}$. Это решение продолжает решение $z_{\gamma_{1}}$ и удовлетворяет неравенству $z_{\gamma_{2}} \triangleleft P_{\gamma_{2}} u$.

Далее повторяем предыдушие построения для продолжения решения $z_{\gamma_{2}}$, затем $z_{\gamma_{3}}$ и т.д. На $n$-м шаге вычисляем $\varphi_{n-1}=\inf \left\{z_{\gamma_{n-1}}, u\right\}$. Выбираем $r=$ $\widetilde{k}\left(\left\|\varphi_{n-1}\right\|+2\left\|z_{\gamma_{n-1}}\right\|+3\right)+1, \varepsilon=1$ и находим $\delta_{n}$, являющееся точной верхней гранищей всевозможных чисел $\delta$, удовлетворяющих условию (12). Пусть $\gamma_{n}=\gamma_{n-1}+$ $\delta_{n} / 2$. Замкнутое множество

$$
\begin{aligned}
U_{n}= & \left\{x_{\gamma_{n}} \in B\left(e_{\gamma_{n}}\right) \mid\left\|x_{\gamma_{n}}\right\| \leqslant \widetilde{k}\left(\left\|\varphi_{n-1}\right\|+2\left\|z_{\gamma_{n-1}}\right\|+3\right)\right. \\
& \left.\wedge x_{\gamma_{n}}(t)=\varphi_{n-1}(t), t \in e_{\gamma_{n-1}}\right\}
\end{aligned}
$$

инвариантно относительно монотонного и предельно монотонно компактного оператора $K_{\gamma_{n}}=\Pi_{\gamma_{n}} K P_{\gamma_{n}}: B\left(e_{\gamma_{n}}\right) \rightarrow B\left(e_{\gamma_{n}}\right)$. Элемент $\varphi_{\gamma_{n}}=\Pi_{\gamma_{n}} \varphi_{n-1} \in U_{n}$ удовлетворяет неравенству $\varphi_{\gamma_{n}} \triangleright K_{\gamma_{n}} \varphi_{\gamma_{n}}$. Таким образом, сушествует локальное решение $z_{\gamma_{n}}$ уравнения (9), определенное на $e_{\gamma_{n}}$, являюшееся наименшим из решений, принадлежащих множеству $U_{n}$. Это решение продолжает решение $z_{\gamma_{n-1}}$ и удовлетворяет неравенству $z_{\gamma_{n}} \triangleleft P_{\gamma_{n}} u$.

Докажем, что, продолжая описанные построения, мы получаем глобальное или предельно продолженное решение. Вычислим $\lim _{i \rightarrow \infty} \gamma_{i}=\eta$ и рассмотрим функцию $z_{\eta}$, определенную на $\bigcup_{i=1}^{\infty} e_{\gamma_{i}}$, сужением которой на $e_{\gamma_{i}}$ будет $z_{\gamma_{i}}, i=1,2, \ldots$. 
Если функция $z_{\eta}$ не является предельно продолженным решением, то найдется такое число $N$, что при всех натуральных $i$ имеет место $\left\|z_{\gamma_{i}}\right\| \leqslant N$. Согласно формуле (8) получаем $\left\|\varphi_{i}\right\| \leqslant \widetilde{k}(N+\|u\|)$. Возьмем $r=\widetilde{k}(\widetilde{k}(N+\|u\|)+2 N+3)+1$, $\varepsilon=1$ и найдем $\bar{\delta}$, являющееся точной верхней границей всевозможных чисел $\delta$, удовлетворяющих условию (12). Таким образом, при любом $i$ выполнено $\delta_{i} \geqslant \bar{\delta}$. Это означает, что за конечное число шагов будет построено глобальное решение уравнения (9).

Пусть полученное здесь решение $z_{\eta}, \eta \leqslant b-a$, не является нижним. Тогда сушествует такое локальное или глобальное решение $\omega_{\gamma}$, что для сужения функции $z_{\eta}$ на $e_{\gamma}$ имеет место $z_{\gamma} \Varangle \omega_{\gamma}$. (Здесь $\gamma<\eta$, если решение $z_{\eta}$ предельно продолженное, и $\gamma \leqslant \eta$, если решение $z_{\eta}$ глобальное.) Вычислим $\Xi=\sup \left\{\xi \mid z_{\xi} \triangleleft \omega_{\xi}\right\}$, где $z_{\xi}, \omega_{\xi}$ - сужения соответствующих функций на $e_{\xi}$. Считаем, что $\Xi=0$, когда множество $\left\{\xi \mid z_{\xi} \triangleleft \omega_{\xi}\right\}$ пусто. Возможны два варианта: 1) $z_{\Xi} \triangleleft \omega_{\Xi}$;2) $z_{\Xi} \Varangle \omega_{\Xi}$. Рассмотрим вначале первый их них.

Найдем такое натуральное $n$, при котором $\Xi \in\left[\gamma_{n-1}, \gamma_{n}\right)$. Обозначим $\varpi_{\gamma}=$ $\inf \left\{z_{\gamma}, \omega_{\gamma}\right\}$. Имеем $\varpi_{\Xi}=z_{\Xi}$. При любом $\xi \in\left(\Xi, \gamma_{n}\right)$ выполнено $\varpi_{\xi} \triangleleft z_{\xi}$ и $\varpi_{\xi} \neq z_{\xi}$. Далее, $K_{\xi} \varpi_{\xi} \triangleleft K_{\xi} z_{\xi}=z_{\xi}, K_{\xi} \varpi_{\xi} \triangleleft K_{\xi} \omega_{\xi}=\omega_{\xi}$ и поэтому $K_{\xi} \varpi_{\xi} \triangleleft \varpi_{\xi}$, $K_{\xi}^{2} \varpi_{\xi} \triangleleft K_{\xi} \varpi_{\xi}$. Для любого положительного $\varepsilon$ вследствие улучшаемости оператора $K$ можно так выбрать $\widehat{\xi} \in\left(\Xi, \gamma_{n}\right)$, что $\left\|K_{\widehat{\xi}} \varpi_{\widehat{\xi}}\right\|-\left\|K_{\Xi} \varpi \Xi\right\| \leqslant \varepsilon$, т.е. $\left\|K_{\widehat{\xi}} \varpi_{\widehat{\xi}}\right\| \leqslant\left\|z_{\Xi}\right\|+\varepsilon$. Вычислим $f_{\gamma_{n}}=\inf \left\{z_{\gamma_{n}}, K_{\widehat{\xi}} \varpi_{\widehat{\xi}}\right\}$. Из формулы (8) получаем

$$
\left\|f_{\gamma_{n}}\right\| \leqslant \widetilde{k}\left(\left\|z_{\gamma_{n}}\right\|+\left\|K_{\widehat{\xi}} \varpi_{\widehat{\xi}}\right\|\right) \leqslant \widetilde{k}\left(2\left\|z_{\gamma_{n}}\right\|+\varepsilon\right) \leqslant \widetilde{k}\left(2\left\|z_{\gamma_{n-1}}\right\|+2+\varepsilon\right) .
$$

Таким образом, если взять $\varepsilon \leqslant 1$, то $f_{\gamma_{n}} \in U_{n}$. Кроме того, так как $K_{\gamma_{n}} f_{\gamma_{n}} \triangleleft$ $K_{\gamma_{n}} z_{\gamma_{n}}=z_{\gamma_{n}}$ и $K_{\widehat{\xi}} f_{\widehat{\xi}} \triangleleft K_{\widehat{\xi}}^{2} \varpi_{\widehat{\xi}} \triangleleft K_{\widehat{\xi}} \varpi_{\widehat{\xi}}$, то $K_{\gamma_{n}} f_{\gamma_{n}} \triangleleft f_{\gamma_{n}}$. Согласно теореме 1 в множестве $U_{n}$ сушествует неподвижная точка $g_{\gamma_{n}}$, для которой $g_{\gamma_{n}} \triangleleft f_{\gamma_{n}} \triangleleft z_{\gamma_{n}}$, причем согласно построениям $g_{\gamma_{n}} \neq z_{\gamma_{n}}$. Это противоречит тому, что $z_{\gamma_{n}}$ является наименьшим из решений, принадлежаших множеству $U_{n}$.

Рассмотрим теперь вторую ситуацию: $z_{\Xi} \Varangle \omega_{\Xi}$. Найдем натуральное $n$, при котором $\Xi \in\left(\gamma_{n-1}, \gamma_{n}\right]$. Обозначим $\varpi_{\gamma}=\inf \left\{z_{\gamma}, \omega_{\gamma}\right\}$. Имеем $\varpi_{\Xi} \triangleleft z_{\Xi}, \varpi_{\Xi} \neq z_{\Xi}$. При любом $\xi \in\left(\gamma_{n-1}, \Xi\right)$ вьполнено $\varpi_{\xi}=z_{\xi}$. Далее, $K_{\Xi} \varpi_{\Xi} \triangleleft K_{\Xi} z_{\Xi}=z_{\Xi}$, $K_{\Xi} \varpi \Xi K_{\Xi} \omega_{\Xi}=\omega_{\Xi}$ и поэтому $K_{\Xi \varpi \Xi \triangleleft \varpi \Xi} \varpi_{\Xi} K_{\Xi}^{2} \varpi_{\Xi} \triangleleft K_{\Xi} \varpi_{\Xi}$. Для любого положительного $\varepsilon$ вследствие улучшаемости оператора $K$ можно так выбрать $\widehat{\xi} \in$ $\left(\gamma_{n-1}, \Xi\right)$, что $\left\|K_{\Xi} \varpi_{\Xi}\right\|-\left\|K_{\widehat{\xi}} \varpi_{\hat{\xi}}\right\| \leqslant \varepsilon$, т.е. $\left\|K_{\Xi} \varpi_{\Xi}\right\| \leqslant\left\|z_{\widehat{\xi}}\right\|+\varepsilon \leqslant\left\|z_{\gamma_{n}}\right\|+\varepsilon$. Вычислим $f_{\gamma_{n}}=\inf \left\{z_{\gamma_{n}}, K_{\Xi} \varpi \Xi\right\}$. Из формулы (8) получаем $\left\|f_{\gamma_{n}}\right\| \leqslant \widetilde{k}\left(\left\|z_{\gamma_{n}}\right\|+\right.$ $\left.\left\|K_{\Xi} \varpi_{\Xi}\right\|\right) \leqslant \widetilde{k}\left(2\left\|z_{\gamma_{n}}\right\|+\varepsilon\right)$. Таким образом, $f_{\gamma_{n}} \in U_{n}$. Кроме того, так как $K_{\gamma_{n}} f_{\gamma_{n}} \triangleleft K_{\gamma_{n}} z_{\gamma_{n}}=z_{\gamma_{n}}$ и $K_{\Xi} f_{\Xi} \triangleleft K_{\Xi}^{2} \varpi_{\Xi} \triangleleft K_{\Xi} \varpi_{\Xi}$, то $K_{\gamma_{n}} f_{\gamma_{n}} \triangleleft f_{\gamma_{n}}$. Согласно теореме 1 в множестве $U_{n}$ сушествует неподвижная точка $g_{\gamma_{n}}$, для которой $g_{\gamma_{n}} \triangleleft$ $f_{\gamma_{n}} \triangleleft z_{\gamma_{n}}$, причем согласно построениям $g_{\gamma_{n}} \neq z_{\gamma_{n}}$. Это противоречит тому, что $z_{\gamma_{n}}$ является наименьшим из решений, принадлежащих множеству $U_{n}$.

Теорема доказана.

СледСТВИЕ. Пусть в банаховом пространстве B, удовлетворяющем $V$-условию, введено отношение порядка, порохденное несплющенным, вольтерровым на системе $v$, вольтеррово сильно миниәдральным и вольтеррово вполне правильным конусом $\Lambda$. Пусть оператор $K: B \rightarrow B$ является улуч- 
шающим, вольтерровым на v, монотонным. Пусть, кроме того, для некоторого әлемента $u \in B$ выполнено неравенство $u \triangleright K u$. Тогда:

- существует такое $\delta>0$ и существует определенное на е л локальное решение $z_{\delta}$ уравнения (9), для которого имеет место оченка $z_{\delta} \triangleleft$ $\Pi_{\delta} u$;

- любое локальное решение $z_{\gamma}$ уравнения (9), для которого имеет место оценка $z_{\gamma} \triangleleft \Pi_{\gamma}$, продолжаемо либо до глобального $z_{b-a}$, удовлетворяющего неравенству $z_{b-a} \triangleleft u$, либо до предельно продолженного решения $z_{\eta}$ такого, что при всех $\delta<\eta$ выполнено $z_{\delta} \triangleleft \Pi_{\delta} u$;

- существует либо нижснее глобальное решение $z_{b-a}$ уравнения (9) такое, что $z_{b-a} \triangleleft и$, либо нижнее предельно продолженное решение $z_{\eta}$, сужение которого $z_{\gamma}$ на любое $e_{\gamma}, \gamma<\eta$, удовлетворяет неравенству $z_{\gamma} \triangleleft \Pi_{\gamma} u$.

Действительно, здесь можно воспользоваться теоремами 4-6, так как из полной правильности конуса $\Lambda$ следует $[17$; гл. $4, \S 33$, п. 33.1, гл. 5, п. 38.2] его нормальность и предельная монотонная компактность монотонного оператора $K: B \rightarrow B$.

Мы уже обращали внимание на то, что во всех рассмотренных утверждениях не предполагается непрерывность оператора $K: B \rightarrow B$. Это позволяет применять полученные здесь результаты, например, к изучению скалярного дифференциального уравнения

$$
x^{\prime}(t)=f(t, x(t)), \quad t \in[a, b]
$$

в котором функция $f:[a, b] \times \mathbb{R} \rightarrow \mathbb{R}$ не удовлетворяет условиям Каратеодори. К таким уравнениям приводят некоторые задачи управления, автоматического регулирования [15], [16]. Классические схемы исследования, использующие непрерывность операторов, к уравнению (16) не применимы. Наиболее "продуктивным" и обшепризнанным является предложенный А.Ф. Филипповым [30] метод, основанньй на построении соответствующего дифференциального включения. В тех случаях, когда уравнение (16) приводится к уравнению с монотонным вольтерровым оператором в некотором банаховом пространстве, можно воспользоваться предложенными вьше утверждениями и исследовать само уравнение, не прибегая к замене его включением.

Будем предполагать, что функция $f$ суммируема по первому аргументу, непрерывна справа по второму аргументу и существует такое $c \in(a, b)$, что при почти всех $t \in[c, b]$ функция $f(t, \cdot)$ не убывает, а при почти всех $t \in[a, c]$ функция $f(t, \cdot)$ не возрастает. Как доказано И.В. Шрагиньм в [31], эти условия обеспечивают измеримость суперпозиции $f(\cdot, x(\cdot))$ для каждой непрерывной функции $x$.

Рассмотрим для уравнения (16) краевую задачу с условием

$$
x(c)=\alpha .
$$

Запишем ее в виде $x^{\prime}(t)=f\left(t, \alpha+\int_{c}^{t} x^{\prime}(s) d s\right)$. Оператор

$$
K: L_{[a, b]} \rightarrow L_{[a, b]}, \quad(K y)(t)=f\left(t, \alpha+\int_{c}^{t} y(s) d s\right),
$$

является улучшающим, вольтерровым на совокупности $v$ множеств $e_{\gamma}=[c-\sigma(\gamma)$, $c+\beta(\gamma)]$, где $\sigma(\cdot), \beta(\cdot)$ - любые неубывающие функции, для которых $\beta(\gamma)-$ $\sigma(\gamma)=\gamma$. Упорядочим пространство $L_{[a, b]}$ конусом неотрицательных функций. 
Этот конус вольтерров на $v$, вольтеррово сильно миниэдральный, вольтеррово вполне правильный и несплющенный. Кроме того, с такой упорядоченностью пространства суммируемых функций оператор $K$ будет монотонным. Все перечисленное позволяет воспользоваться следствием к теореме 6 и утверждать, что если для некоторого $u \in D_{[a, b]}$ въполнено неравенство $u^{\prime}(t) \geqslant f(t, u(t)), t \in[a, b]$, $u(c) \geqslant \alpha, m o:$

- существует такое $\delta>0$ и существует определенное на $e_{\delta}=[c-\eta(\delta)$, $c+\beta(\delta)]$ локальное решение $x_{\delta}$ задачи $(16),(17)$, для которого имеет место оченка $x_{\delta}^{\prime}(t) \leqslant u^{\prime}(t), \quad t \in e_{\delta}$

- любое локальное решение $x_{\gamma}$ задачи (16), (17), для которого имеет место оченка $x_{\gamma}^{\prime}(t) \leqslant u^{\prime}(t), \quad t \in e_{\gamma}$, продолжаемо до решения $x_{\xi}$, определенного на таком интервале $\left(\xi_{1}, \xi_{2}\right)$, что

$$
\xi_{1}=a \vee \int_{\xi_{1}}^{c}\left|x_{\xi}^{\prime}(s)\right| d s=\infty, \quad \xi_{2}=b \vee \int_{c}^{\xi_{2}}\left|x_{\xi}^{\prime}(s)\right| d s=\infty
$$

и удовлетворяющего неравенству $x_{\xi}^{\prime}(t) \leqslant u^{\prime}(t), t \in\left(\xi_{1}, \xi_{2}\right)$;

- существует нижнее решение $\underline{x}_{\xi}$ задачи (16), (17), определенное на таком интервале $\left(\xi_{1}, \xi_{2}\right)$, что

$$
\xi_{1}=a \vee \int_{\xi_{1}}^{c}\left|\underline{x}_{\xi}^{\prime}(s)\right| d s=\infty, \quad \xi_{2}=b \vee \int_{c}^{\xi_{2}}\left|\underline{x}_{\xi}^{\prime}(s)\right| d s=\infty
$$

удовлетворяющее неравенству $\underline{x}_{\xi}^{\prime}(t) \leqslant u^{\prime}(t), t \in\left(\xi_{1}, \xi_{2}\right) ;$ кроме того, для любого локального решения $x_{\gamma}$ задачи (16), (17), определенного на $e_{\gamma} \subset\left(\xi_{1}, \xi_{2}\right)$, выполнено $\underline{x}_{\xi}^{\prime}(t) \leqslant x_{\gamma}^{\prime}(t), t \in e_{\gamma}$.

Это утверждение применимо также, если при почти всех $t \in[a, b]$ функция $f(t, \cdot)$ не убывает. В этом случае следует взять $c=a$. Если при почти всех $t \in[a, b]$ функция $f(t, \cdot)$ не возрастает, то утверждение также верно при $c=b$.

Автор благодарит А.И. Булгакова за критику и полезные советы и всех участников Тамбовского семинара за обсуждение результатов этой работы.

\section{Список литературы}

1. Чапльгин C. A. Новый метод приближенного интегрирования диффференциальных уравнений. М.-Л.: Гостехиздат, 1950.

2. Азбелев Н. В. О границах применимости теоремы Чаплыгина о диффференциальных неравенствах // Матем. сб. 1956. Т. 39 (81). С. 161-178.

3. Азбелев Н. В., Цалюк З. Б. Об интегральных неравенствах // Матем. сб. 1962. Т. 56 (98). C. $325-342$.

4. Курпель Н. С., Шувар Б. А. Двусторонние операторные неравенства и их применения. Киев: Наукова думка, 1980.

5. Азбелев Н.В., Максимов В. П., Рахматуллина Л. Ф. Введение в теорию функционально-дифференциальных уравнений. М.: Наука, 1991.

6. Азбелев Н.В., Максимов В. П., Рахматуллина Л. Ф. Элементы современной теории функционально-дифференциальных уравнений. Методы и приложения. М.: Институт компьютерных исследований, 2002.

7. Азбелев Н.В., Рахматуллина Л. Ф. К вопросу о функционально-дифференциальных неравенствах и монотонных операторах // Функционально-дифференциальные уравнения. Пермь: Изд-во Пермского политехн. ин-та, 1986. С. 3-9. 
8. Жуковский E. C. Об интегральных неравенствах в пространствах суммируемых функций // Диффференц. уравнения. 1982. Т. 18. №4. С. 580-584.

9. Жуковская Т. В., Жуковский Е. С. Монотонные операторы и разрешимость функционально-дифференциальных уравнений // Вестн. Тамбовского ун-та. Сер. естеств. и техн. науки. Т. 3. № 2. Тамбов: Изд-во ТГУ, 1998. С. 171-176.

10. Шиндяпин А.И. О краевой задаче для одного сингулярного уравнения // Дифференц. уравнения. 1984. Т. 20. № 3. С. 450-455.

11. Анохин А. В. О линейных импульсных системах для функционально-дифференциальных уравнений // Докл. АН СССР. 1986. Т. 28. № 5. С. 1037-1040.

12. Румлниев A.H. Исследование разрешимости краевых задач с применением векторных априорных неравенств при наличии импулсных возмущений // Изв. вузов. Сер. матем. 1996. №6. C. $75-82$.

13. Гусаренко C.A., Жуковский Е. С., Максимов В.П. К теории функционально-дифференциальных уравнений с локально волтерровыми операторами // Докл. АН СССР. 1986. Т. 287. № 2. С. 268-272.

14. Драйвер Р. Д. Топологии для уравнений нейтрального типа и классическая электродинамика // Дифференциальные уравнения с отклоняющимся аргументом. Киев: Наукова думка, 1977. С. 113-127.

15. Берщанский $Я$. $M$. Траектории линейных систем с нелинейностью типа реле // Автоматика и телемеханика. 1982. № 7. С. 19-27.

16. Фуллер A. T. Оптимизация релейных систем регулирования по различным критериям качества // Теория дискретных оптимальных и самонастраивающихся систем. Труды І конгресса ИФАК. М.: Изд-во АН СССР, 1961. С. 584-605.

17. Красносельский М. А., Забрейко П. П. Геометрические методы нелинейного анализа. М.: Наука, 1975.

18. Канторович Л. В., Ажилов Г. П. Функциональный анализ. М.: Наука, 1984.

19. Тихонов $A$. Н. О функциональных уравнениях типа Вольтерра и их применениях к некоторым задачам математической физики // Бюл. Моск. ун-та. Секц. А. 1938. Т. 1. № 8. C. $1-25$.

20. Бродский $M . C$. Треугольные и жордановы представления линейных операторов. М.: Наука, 1969.

21. Бухгейм А. Л. Уравнения Вольтерра и обратные задачи. Новосибирск: Наука, 1983.

22. Гохберг И.Ц., Крейн М.Г. Теория вольтерровых операторов в гильбертовом пространстве и ее приложения. М.: Наука, 1967.

23. Гусаренко С.А. Об одном обобщении понятия вольтеррова оператора // Докл. АН CCCP. 1987. T. 295. № 5. С. 1046-1049.

24. Жуковский Е. С. К теории уравнений Вольтерра // Дифференц. уравнения. 1989. Т. 25. №9. C. 1599-1605.

25. Жуковский E. C. Вольтерровость и спектральные свойства оператора внутренней суперпозиции // Дифференц. уравнения. 1994. Т. 30. № 2. С. 147-149.

26. Жуковский $E$. C. Линейные эволюционные функционально-дифференциальные уравнения в банаховом пространстве. Тамбов: Изд-во ТГУ, 2003.

27. Курбатов В.Г. Линейные дифференциально-разностные уравнения. Воронеж: Изд-во ВГУ, 1990.

28. Ливиии M. С. О спектральном разложении линейных несамосопряженных операторов // Матем. сб. 1954. Т. 34 (76). С. 145-198.

29. Сумин В.И. Функционально-операторные вольтерровы уравнения в теории оптимального управления распределенньми системами // Докл. АН СССР. 1989. Т. 305. № 5 . C. $1056-1059$.

30. Филиппов A. Ф. Дифференциальные уравнения с разрывной правой частью // Матем. сб. 1960 . Т. 51 (93). С. 99-128.

31. Шрагин И. В. Измеряемость суперпозиций разрывных функций // Труды Тамб. ин-та хим. машиностроения. 1969. С. 6-8.

Тамбовский государственный университет, г. Тамбов

E-mail: aib@tsu.tmb.ru 